\title{
Desmoplastic Neurotropic Melanoma
}

National Cancer Institute

\section{Source}

National Cancer Institute. Desmoplastic Neurotropic Melanoma. NCI Thesaurus. Code C48614.

A desmoplastic melanoma characterized by the presence of nerve infiltration by atypical spindled melanocytes. 